\title{
Modification of an SiO2(Au)/Si Surface by Irradiation with Argon Ions
}

\author{
V. D. Bundyukova ${ }^{1,2^{*}}$, D. V. Yakimchuk ${ }^{1}$, E. Yu. Kaniukov ${ }^{3}$, D. I. Tishkevich ${ }^{1,4}$, M. D. Kutuzau ${ }^{1}$, \\ V. V. Prigodich ${ }^{1}$, A. A. Shemukhin ${ }^{1,5}$, Yu. V. Balakshin ${ }^{1,5}$, A. V. Nazarov ${ }^{2}$, A. V. Kozhemiako ${ }^{6}$,
} A. P. Evseev ${ }^{2,6}$, and A. E. Ieshkin ${ }^{6}$

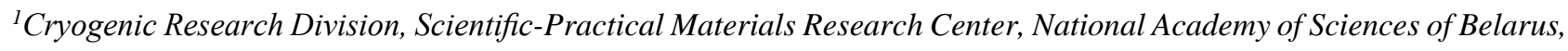
Minsk, 220072 Belarus

${ }^{2}$ Institute of Nuclear Physics, Moscow State University, Moscow, 119991 Russia

${ }^{3}$ National University of Science and Technology MISIS, Moscow, 119049 Russia

${ }^{4}$ South Ural State University, Chelyabinsk, 454021 Russia

${ }^{5}$ Center for Quantum Technologies, Moscow State University, Moscow, 119991 Russia

${ }^{6}$ Department of Physics, Moscow State University, Moscow, 119991 Russia

"E-mail: victoria.bundyukova@gmail.com

Abstract: This article presents the results of gold deposition into pores of $\mathrm{SiO}_{2} / \mathrm{Si}$ matrices and the modifications of the obtained $\mathrm{SiO}_{2}(\mathrm{Au}) / \mathrm{Si}$ systems by irradiation with argon $\mathrm{Ar}^{+}$ions with an energy of $100 \mathrm{keV}$ with a fluence of $5 \times 10^{14} \mathrm{ion} / \mathrm{cm}^{2}$. The effect of irradiation on changes in the surface topography of $\mathrm{SiO}_{2}(\mathrm{Au}) / \mathrm{Si}$ systems and the signal intensity of surface-enhanced Raman scattering during the detection of the model analyte (methylene blue) have been shown.

Keywords: porous matrices, template synthesis, gold nanostructures, surface modification, ion irradiation, defect formation, surface-enhanced Raman scattering.

\section{INTRODUCTION}

Localized surface plasmons are formed as a result of exposure of metallic nanostructures of copper, silver, and gold to visible electromagnetic radiation [1,2]. This increases the strength of the electrical field at the surface of metallic nanostructures [3]. The increase in surface roughness on a nanometer scale leads to the increased strength of the resulting field. This effect is used to improve the sensitivity of such spectrometric methods as fluorescence spectroscopy and Raman scattering. The increase in the Raman scattering cross-section of the investigated substances is so significant that this technique is called surface-enhanced Raman scattering (SERS). In some cases, the SERS method can reach the sensitivity required to detect analytes with monomolecular concentrations [4]. Taking the results 
achieved in the production of SERS active substrates and a wide range of applications in biology, medicine, and sensorics [5-7] into account, the search for effective and inexpensive SERS active substrates is still in progress.

Previously, a method was proposed to create nanostructured plasmon-active surfaces from copper and silver by template synthesis in $\mathrm{SiO}_{2} / \mathrm{Si}$ ion-track matrices using chemical deposition of metal into matrix pores $[8,9]$. It was shown that it is possible to control the morphology of structures in order to create the highest roughness of structures at the nano level by changing the conditions of deposition (electrolyte concentration and temperature as well as the size of matrix pores) and the effectiveness of such structures for SERS was demonstrated [11, 12]. Despite the undoubted advantages of nanostructures based on silver and copper, they have one significant disadvantage, i.e., severe corrosion in air and with water analytes. Gold nanostructures have no such disadvantage [2, 13].

It is possible to increase the rate of gain using the directed modification of the surface of plasmonactive nanostructures by ion beam irradiation. This process changes the surface topography and under certain conditions leads to a more developed surface topography [14-16]. Such self-organized restructuring of the topography under intense ionization radiation can cause the appearance of a number of new interesting effects that will influence the SERS signal intensity when detecting various chemical and biological objects.

This article presents a method for obtaining SERS active substrates based on gold nanoparticles in the pores of $\mathrm{SiO}_{2} / \mathrm{Si}$ matrices and a method for their modification by irradiation with argon ions. The methods of scanning electron microscopy (SEM) and energy dispersion analysis (EDA) were used to examine the structural and morphological characteristics of the obtained samples. The efficiency of the newly obtained SERS substrates was demonstrated by detection of the model analyte with concentrations up to $10^{-4} \mathrm{M}$.

\section{MATERIALS AND METHODS}

Porous $\mathrm{SiO}_{2} / \mathrm{Si}$ matrices with pore diameters of $~ 350$ and pore density of $5 \times 10^{7} \mathrm{~cm}^{-2}$ were used for gold deposition. The process of obtaining such matrices was described in detail in our work [17]. Features of control of the pore parameters of $\mathrm{SiO}_{2} / \mathrm{Si}$ matrices were described in [18]. Gold structures were formed in the porous $\mathrm{SiO}_{2} / \mathrm{Si}$ matrix by electrodeless deposition, when the substrates were immersed in an aqueous solution of $0.01 \mathrm{M} \mathrm{AuCl}_{3} \times \mathrm{H}_{2} \mathrm{O}$ and $5 \mathrm{M} \mathrm{HF}$ in the volume ratio of 1:1. After deposition, the samples were washed several times in distilled water and dried at room temperature. Primary validation of the deposition results was carried out on a scanning electron microscope (SEM, Hitachi TM3030) with an attachment for energy dispersive X-ray spectroscopy (EDX, Bruker XFlash 
MIN SVE). A detailed morphology analysis of gold nanostructures was performed on a JEOL JSM7500F SEM.

The samples were irradiated with $\mathrm{Ar}^{+}$ions with an energy of $100 \mathrm{keV}$ at the HVEE-500 heavy ion accelerator complex with energies up to $500 \mathrm{keV}$ [19-21]. The irradiation was carried out at an angle of $7^{\circ}$ with respect to normal to the sample surface. The fluence of the irradiation was $5 \times 10^{14} \mathrm{ion} / \mathrm{cm}^{2}$. The interaction of $\mathrm{Ar}^{+}$ions with gold nanostructures was modeled in the SRIM program [22] by Monte Carlo methods in the approximation of binary collisions. The energy of $\mathrm{Ar}^{+}$ions was $100 \mathrm{keV}$, the fluence of irradiation was $5 \times 10^{14} \mathrm{ion} / \mathrm{cm}^{2}$. Gold was used as a target. The Au model target had the following parameters: the density of $19.31 \mathrm{~g} / \mathrm{cm}^{3}$, the atomic density of $5.9 \times 10^{22} \mathrm{at} / \mathrm{cm}^{3}$, and the displacement energy $\left(E_{\text {disp }}\right)$ of $25 \mathrm{eV}$. Defect formation was modeled within the Norgett-RobinsonTorrence model (NRT) [23].

Surface-enhanced Raman scattering on gold structures was studied on the MB model analyte. The research was carried out using a spectrometer made by Sol Instruments. Light was focused through a $40 \times$ lens on the sample; the Raman scattering signal was collected using the same lens. The laser power on the sample surface was $1 \mathrm{~mW}$ and the signal collection time was $1 \mathrm{~s}$.

\section{RESULTS AND DISCUSSION}

Figs. 1a,b show typical SEM images of gold nanostructures grown in the pores of the $\mathrm{SiO}_{2} / \mathrm{Si}$ template by electrodeless deposition. The selected pore diameter in the $\mathrm{SiO}_{2}$ matrix layer $(\sim 350 \mathrm{~nm})$ provided the formation of individual metal-lic nanostructures of a complex shape with diameters of 400-500 $\mathrm{nm}$ and with sharp crystal faces with characteristic sizes of less than $50 \mathrm{~nm}$. Energy dispersive spectroscopy (EDS) analysis confirmed that structures of an arbitrary shape on the dielectric surface consist of gold (Fig. 1e). In addition to the signal from gold, the spectra contain lines of oxygen and silicon related to the matrix material. The similar morphology of the structures within one substrate makes it possible to use them for further studies.

In order to increase the surface roughness of gold nanostructures, they were irradiated with $\mathrm{Ar}^{+}$ions. The results of SEM research of gold structures in the pores of the $\mathrm{SiO}_{2} / \mathrm{Si}$ matrix after irradiation are shown in Figs. 1c,d. 

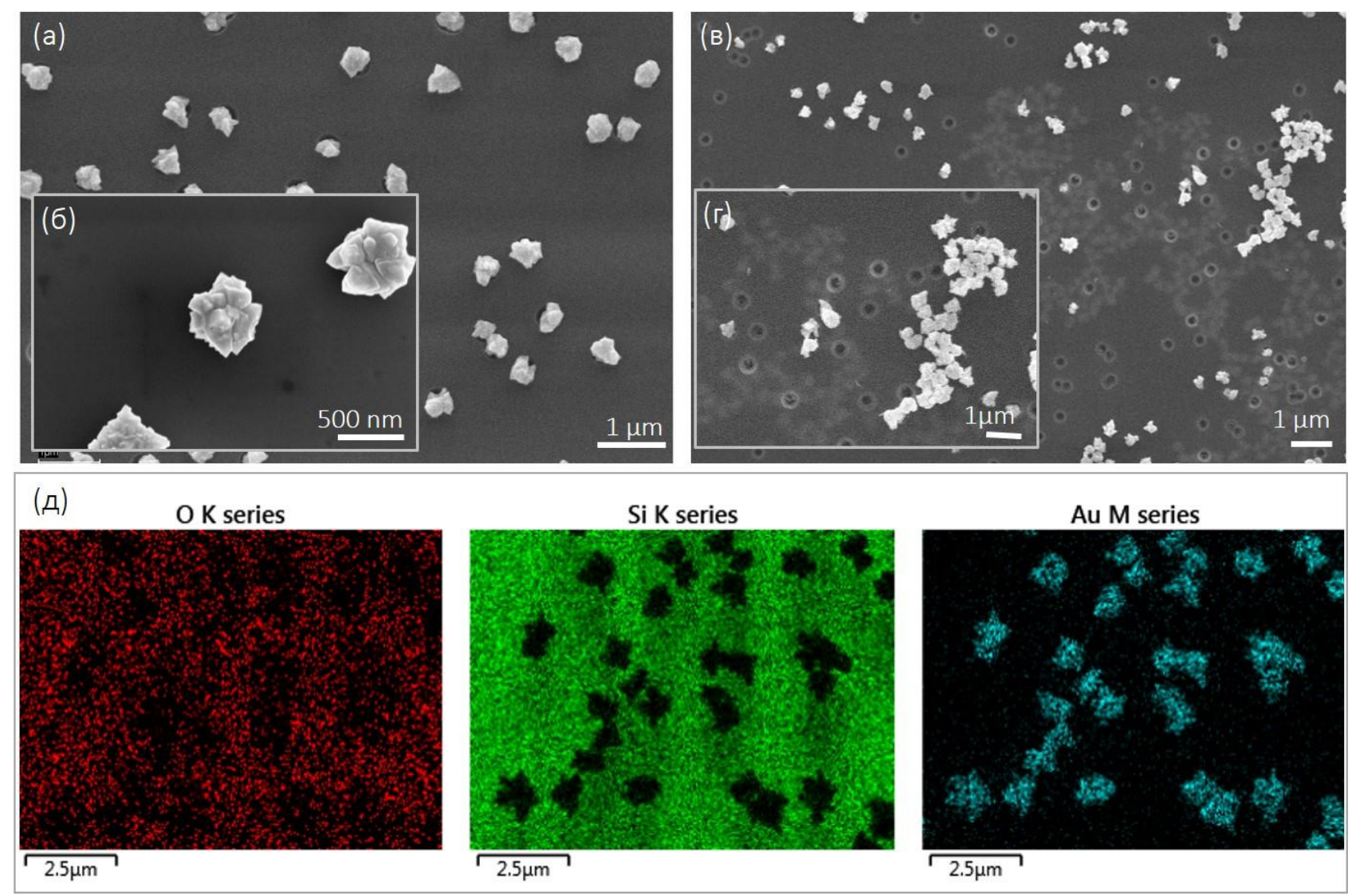

Figure 1. SEM image of gold nanostructures in the porous $\mathrm{SiO}_{2} / \mathrm{Si}$ matrix before (а), (б) and after (в), (г) irradiation as well as the EDS map (д) corresponding to the SEM image (а).

Figure 2 shows depth distributions of the atomic concentration of embedded argon ions in gold and vacancies in the gold target for irradiation with $\mathrm{Ar}^{+}$ions with the energy of $100 \mathrm{keV}$ calculated according to the NRT model. Modeling in SRIM was carried out under the assumption of zero target temperature in the absence of atom oscillations. During irradiation at room temperature, selfannealing of defects will occur [24]. Therefore, the presented estimations are overstated. The calculated average projective range of the argon ion was $37 \mathrm{~nm}$, and with effort $20 \mathrm{~nm}$. According to the modeling, the distribution of the impurities begins on the surface of the sample and reaches $1.5 \times$ $10^{19} \mathrm{ion} / \mathrm{cm}^{3}$ at a depth of $20-50 \mathrm{~nm}$. The defect distribution profile also starts from the surface with the maximum defect distribution at depths of 15-30 nm. According to the modeling, there is a positive asymmetry characteristic of slow ions of medium and large masses for the distribution of vacancies. 


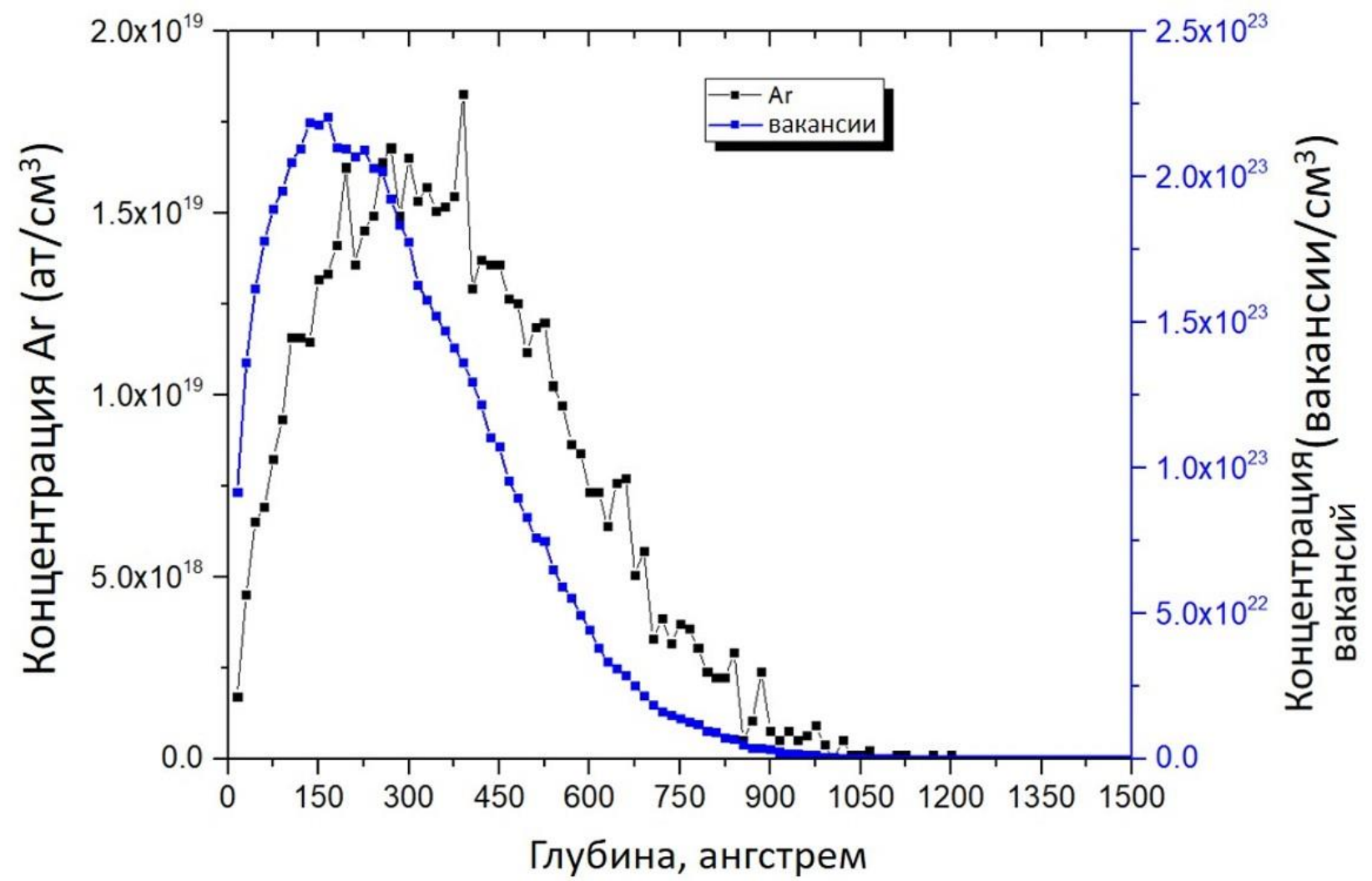

Figure 2. The calculated values of the distribution of embedded argon ions (black) in comparison with the distribution of vacancies in the gold target (blue) for irradiation with $\mathrm{Ar}^{+}$ions with the energy of $100 \mathrm{keV}$.

The comparison of SEM images of the $\mathrm{SiO}_{2}(\mathrm{Au}) / \mathrm{Si}$ system before (Figs. 1a,b) and after (Figs. 1c,d) irradiation by $\mathrm{Ar}^{+}$ions shows that the effect of accelerated particles with energies of $100 \mathrm{keV}$ may accidentally push out metal deposit from the pores of the silicon oxide matrix with the subsequent agglomeration of gold crystals resulting in the formation of metal accumulations with an area of $\sim 2-3$ $\mu \mathrm{m}^{2}$.

The yield of metal deposits from the pores of the silicon oxide matrix may be related to the accumulation of charge in the intensively bombarded metallic nanostructures localized in the pores of the dielectric matrix. The metal-semiconductor contact at the pore bottom area does not provide timely charge drain due to the formation of the Schottky barrier on the metal-semiconductor boundary [25]. Intensive irradiation leads to electrostatic repulsion of a charged metal nanostructure from the pore of oxide matrix, which causes the formation of conglomerates on the surface of the $\mathrm{SiO}_{2}$. Experiments on irradiation of the $\mathrm{SiO}_{2}(\mathrm{Au}) / \mathrm{Si}$ system with different doses and intensities of irradiation in the future will allow us to determine the threshold value of the charge at which nanostructures are pushed out from the pores of the oxide matrix.

We note that the conglomeration of gold crystals should lead to an increase in the efficiency of SERS of the investigated samples due to the closeness of nanoscale faces of crystallites with respect to each 
other. The latter is accompanied by the formation of so-called "hot spots", areas in which the electric field strength increases to a much higher level than on the isolated structure. This assumption was tested in the SERS research of the MB model analyte with a concentration of $10^{-4} \mathrm{~mol} / \mathrm{L}$. Figure 3 shows the MB spectra obtained on substrates containing gold nanostructures before and after irradiation.

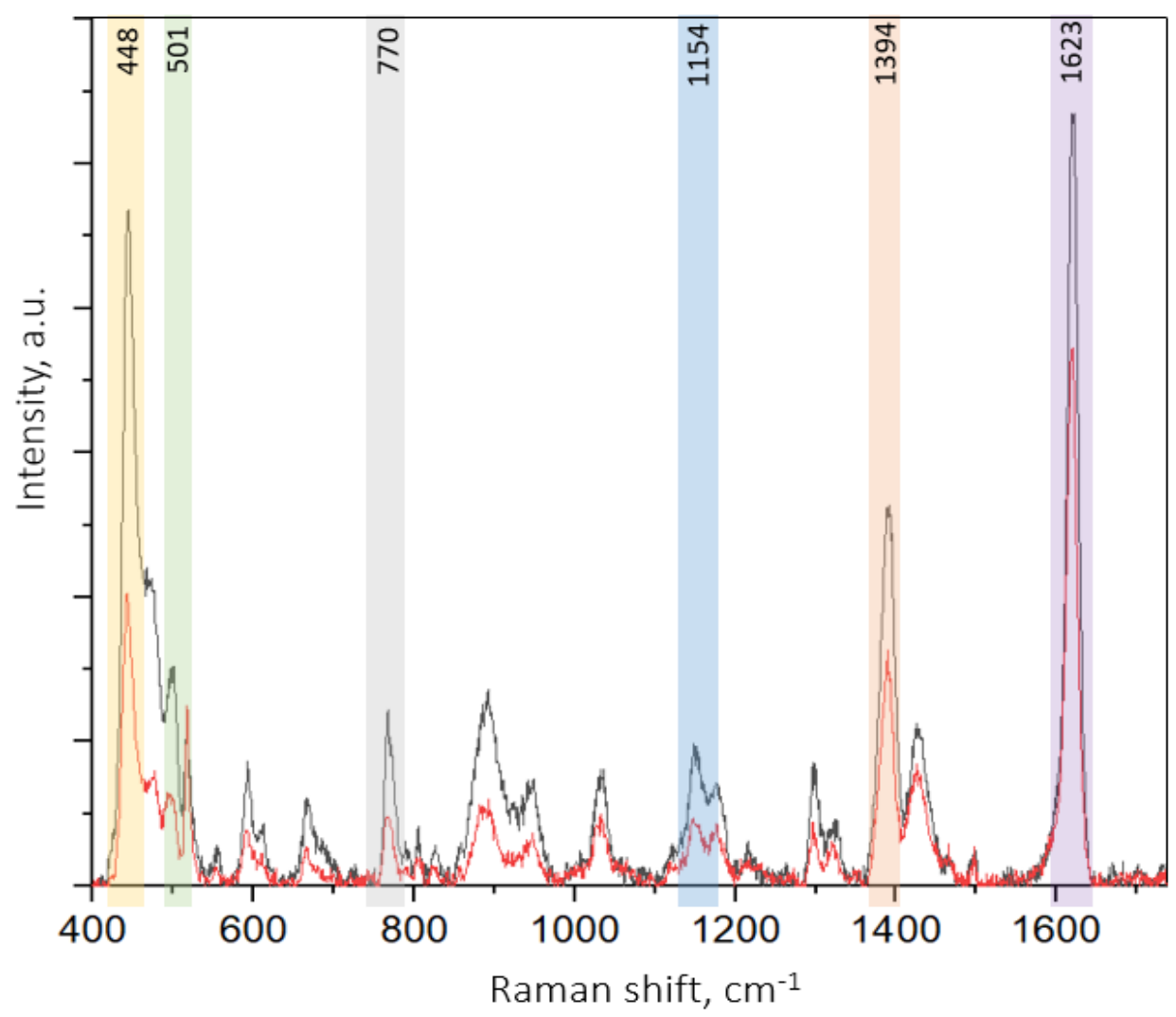

Figure 3. The SERS spectra of the MB analyte with a concentration of $10^{-4}$ mol/L obtained on structures before (red) and after (black) irradiation.

SERS spectra were obtained by averaging five spectra taken at different locations of metal structures within a single SERS substrate. In the spectra, the 448, 501, 770,1154, 1394, and $1623 \mathrm{~cm}^{-1}$ peaks, which correspond to the MB main Raman lines, can be clearly seen [26]. Characteristic 448 and 1623 $\mathrm{cm}^{-1} \mathrm{MB}$ peaks, which correspond to $\mathrm{C}-\mathrm{C}$ and $\mathrm{C}-\mathrm{N}-\mathrm{C}$ oscillations of SERS spectra have high intensities, which indicates good adsorption of molecules on the substrates. SERS spectra obtained on irradiated samples, i.e., taken in the area of accu-mulation of gold structures, are superior in intensity to spectra obtained from isolated gold structures by up to $\sim 80 \%$.

Therefore, the primary experiments to modify the surface of gold nanostructures in the pores of iontrack matrices did not exhibit the desired effect, while the irradiated samples showed better efficiency 
in SERS measurements compared to native samples. It is assumed that it will be possible to modify the gold surface using other irradiation modes, which will be done in future research.

\section{CONCLUSIONS}

The methods of electrodeless deposition were used to form gold structures with nanosize faces in the pores of an $\mathrm{SiO}_{2} / \mathrm{Si}$ template. Under the action of irradiation with argon ions with an energy of 100 $\mathrm{keV}$ and a fluence of $5 \times 10^{14} \mathrm{ion} / \mathrm{cm}^{2}$, gold nanostructures were pushed out of the pores of the $\mathrm{SiO}_{2} / \mathrm{Si}$ matrix and formed conglomerates of a complex shape. SERS analyses of unirradiated and irradiated samples showed that the latter make it possible to in-crease the intensity of Raman scattering from the model analyte by $80 \%$ in comparison with SERS spectra obtained on native samples.

\section{ACKNOWLEDGMENTS}

This work was supported by the project H2020-MSCA-RISE-2017-778308SPINMULTIFILM; the scientific and technical program Technology-SG [project № 3.1.5.1]; the Ministry of Education and Science of the Russian Federation within the Competitiveness Enhancement Program NUST “MISIS” (№ K-2018-036), implemented by the Government Decree of 16 March 2013 № 211 and the Russian Foundation for Basic Research [project № 19-32-50058].

\section{REFERENCES}

[1] B. H. Nguyen, V. H. Nguyen, and H. N. Tran, Adv. Nat. Sci. Nanosci. Nanotechnol. 7, 033001 (2016). doi $10.1088 / 2043-6262 / 7 / 3 / 033001$

[2] D. Yakimchuk, E. Kaniukov, V. Bundyukova, A. Shumskaya, M. Kutuzau, S. Demaynov, and V. Sivakov, in 2018 IEEE 8th International Conference Nanomaterials: Application \& Properties (NAP), 2018, 1-4, doi 10.1109/NAP.2018.8914837

[3] M. R. Gonçalves, J. Phys. D. Appl. Phys. 47, 1-44 (2014). doi 10.1088/0022-3727/47/21/213001

[4] H. Guo, K. Qian, A. Cai, J. Tang, and J. Liu, Sensors Actuators B Chem. 300, 126846 (2019). doi 10.1016/j.snb.2019.126846

[5] Almehmadi, L. M., Curley, S. M., Tokranova, N. A., Tenenbaum, S. A., \& Lednev, I. K. Scientific Reports, 9(1) (2019). doi:10.1038/s41598-019-48650-y.

[6] Mao, P., Liu, C., Favraud, G., Chen, Q., Han, M., Fratalocchi, A., \& Zhang, S. Nature Communications, 9(1). (2018). doi:10.1038/s41467-018-07869-5

[7] Patra, P. P., Chikkaraddy, R., Tripathi, R. P. N., Dasgupta, A., \& Kumar, G. V. P. Nature Communications, 5(1). (2014). doi:10.1038/ncomms5357

[8] Z. Lin and L. He, Curr. Opin. Food Sci. 28, 82-87 (2019). doi 10.1016/j.cofs.2019.10.001

[9] X. Pan, L. Li, H. Lin, J. Tan, H. Wang, M. Liao, C. Chen, B. Shan, Y. Chen, and M. Li, Biosens. Bioelectron. 145, 111713 (2019). doi 10.1016/j.bios.2019.111713 
[10] Y. Wang, S. Zong, N. Li, Z. Wang, B. Chen, and Y. Cui, Nanoscale 11, 2460-2467 (2019). doi 10.1039/C8NR06929H

[11] V. Sivakov, E. Y. Kaniukov, A. V. Petrov, O. V. Korolik, A. V. Mazanik, A. Bochmann, S. Teichert, I. J. Hidi, A. Schleusener, D. Cialla, M. Eugenia Toimil-Molares, C. Trautmann, J. Popp, and S. E. Demyanov, J. Cryst. Growth 400, 21-26 (2014). doi 10.1016/j.jcrysgro.2014.04.024

[12] D. V. Yakimchuk, E. Y. Kaniukov, S. Lepeshov, V. D. Bundyukova, S. E. Demyanov, G. M. Arzumanyanm, N. V. Doroshkevich, K. Z. Mamatkulov, A. Bochmann, M. Presselt, O. Stranik, S. A. Khubezhov, A. E. Krasnok, A. Alù, and V. A. Sivakov, J. Appl. Phys. 126, 233105 (2019). doi 10.1063/1.5129207

[13] Zhang, W., Li, C., Gao, K., Lu, F., Liu, M., Li, X., ... Zhao, J. Nanotechnology, 29(20), 205301 (2018). doi:10.1088/1361-6528/aab294

[14] A.E. Ieshkin, D.S. Kireev, A.A. Tatarintsev, V.S. Chernysh, Nucl. Instruments Methods Phys. Res. Sect. B Beam Interact. with Mater. Atoms 460, 165-168 (2019). doi 10.1016/j.nimb.2019.03.054

[15] N.N. Andrianova, A.M. Borisov, E.S. Mashkova, A.A. Shemukhin, V.I. Shulga, Yu. S. Virgiliev, Nucl. Instruments Methods Phys. Res. Sect. B Beam Interact. with Mater. Atoms 354, 146-150 (2015). doi 10.1016/j.nimb.2014.11.071

[16] A.V. Kozhemiako, A.P. Evseev, Yu.V. Balakshin, A.A. Shemukhin, Semiconductors 53 (6), $800-805$ (2019), doi 10.1134/S1063782619060095

[17] E. Y. Kaniukov, J. Ustarroz, D. V Yakimchuk, M. Petrova, H. Terryn, V. Sivakov, and A. V Petrov, Nanotechnology 27, 115305 (2016). doi 10.1088/0957-4484/27/11/115305

[18] V. Bundyukova, D. Yakimchuk, E. Shumskaya, A. Smirnov, M. Yarmolich, and E. Kaniukov, Mater. Today Proc. 7, 828-834 (2019). doi 10.1016/j.matpr.2018.12.081

[19] Yu V. Balakshin, A.A. Shemukhin, A.V. Nazarov, V.A. Kozhemiako, V.S. Chernysh, Technical Physics 63 (12), 1861-1867 (2018), doi 10.1134/S106378421812023X

[20] Yu.V. Balakshin, A.V. Kozhemiako, S. Petrovic, M. Erich, A.A. Shemukhin, V.S. Chernysh, Semiconductors 53 (8), 1011-1017 (2019), doi 10.1134/S1063782619080062

[21] A.A. Shemukhin, Yu.V. Balakshin, P.N. Chernykh, V.S. Chernysh, Journal of Surface Investigation: X-ray, Synchrotron and Neutron Techniques 7, 318-321 (2013). doi 10.1134/S1027451013020456

[22] J. F. Ziegler, M. D. Ziegler, and J. P. Biersack, Nucl. Instruments Methods Phys. Res. Sect. B Beam Interact. with Mater. Atoms 268, 1818-1823 (2010). doi 10.1016/j.nimb.2010.02.091

[23] K. Nordlund, S.J. Zinkle, A.E. Sand, F. Grandberg, R.S> Averback, R.E. Stoller, T. Suzudo, L. Malerba, F. Banhart, W.J. Weber, F. Willaime, S.L. Dudarev, D. Simeone, J. of Nucl. Mat. 512, 450-479 (2018), doi 10.1016/j.jnucmat.2018.10.027

[24] A.A. Shemukhin, Yu.V. Balakshin, A.P. Evseev, V.S. Chernysh, Nucl. Instruments Methods Phys. Res. Sect. B Beam Interact. with Mater. Atoms 406, 507-510 (2017), doi 10.1016/j.nimb.2017.04.055

[25] E. Y. Kaniukov, D. V. Yakimchuk, V. D. Bundyukova, A. E. Shumskaya, A. A. Amirov, and S. E. Demyanov, Adv. Condens. Matter Phys. 2018, 1-8 (2018). doi 10.1155/2018/4927829

[26] G.-N. Xiao and S.-Q. Man, Chem. Phys. Lett. 447, 305-309 (2007). doi 10.1016/j.cplett.2007.09.045 